\title{
More relaxed supersymmetric electroweak baryogenesis
}

\author{
A. Riotto* \\ CERN, TH Division, CH-1211 Geneva 23, Switzerland \\ (Received 12 May 1998; published 2 October 1998)
}

\begin{abstract}
We reanalyze the issue of the generation of baryon asymmetry at the electroweak phase transition in the MSSM and compute the baryon asymmetry assuming the presence of nontrivial $C P$-violating phases in the parameters associated with the left-right top squark mixing term and the Higgsino mass $\mu$. Making use of the closed time-path formalism of nonequilibrium field theory, we write down a set of quantum Boltzmann equations describing the local number density asymmetries of the particles involved in supersymmetric electroweak baryogenesis. $C P$-violating sources manifest "memory" effects which are typical of the quantum transport theory and are not present in the classical approach. Compared to previous estimates, these nonMarkovian features enhance the final baryon asymmetry by at least two orders of magnitude. This means that a $C P$-violating phase $\left|\sin \phi_{\mu}\right|$ as small as $10^{-3}$ (or even smaller) is enough to generate the observed baryon asymmetry. [S0556-2821(98)08221-6]
\end{abstract}

PACS number(s): 12.60.Jv, 98.80.Cq

\section{INTRODUCTION AND SUMMARY}

The presence of unsuppressed baryon-number-violating processes at high temperatures within the standard model (SM) of weak interactions makes the generation of the baryon number at the electroweak scale an appealing scenario [1]. The baryon-number-violating processes also impose severe constraints on models where the baryon asymmetry is created at energy scales much higher than the electroweak scale [2]. Unfortunately, the electroweak phase transition is too weak in the SM [3]. This means that the baryon asymmetry generated during the transition would subsequently be erased by unsuppressed sphaleron transitions in the broken phase. The most promising and wellmotivated framework for electroweak baryogenesis beyond the SM seems to be supersymmetry (SUSY). Electroweak baryogenesis in the framework of the minimal supersymmetric standard model (MSSM) has attracted much attention in recent years, with particular emphasis on the strength of the phase transition [4-6] and the mechanism of baryon number generation [7-10].

Recent analytical [11,12] and lattice computations [13] have revealed that the phase transition can be sufficiently strongly first order if the ratio of the vacuum expectation values of the two neutral Higgs bosons $\tan \beta$ is smaller than $\sim 4$. Moreover, taking into account all the experimental bounds as well as those coming from the requirement of avoiding dangerous color breaking minima, the lightest Higgs boson should be lighter than about $105 \mathrm{GeV}$, while the right-handed top squark mass might be close to the present experimental bound and should be smaller than, or of the order of, the top quark mass [12].

Moreover, the MSSM contains additional sources of $C P$ violation in addition to the Cabibbo-Kobayashi-Maskawa (CKM) matrix phase. These new phases are essential for the

\footnotetext{
*On leave of absence from Department of Physics, Theoretical Physics, University of Oxford, U.K. Email address: riotto@nxth04.cern.ch.
}

generation of the baryon number since large $C P$-violating sources may be locally induced by the passage of the bubble wall separating the broken from the unbroken phase during the electroweak phase transition. Baryogenesis is fueled when transport properties allow the $C P$-violating charges to efficiently diffuse in front of the advancing bubble wall where anomalous electroweak baryon-violating processes are not suppressed [14]. The new phases appear in the soft supersymmetry breaking parameters associated with the top squark mixing angle and with the gaugino and neutralino mass matrices; large values of the top squark mixing angle are, however, strongly restricted in order to preserve a sufficiently strong first order electroweak phase transition. Therefore, an acceptable baryon asymmetry from the top squark sector may only be generated through a delicate balance between the values of the different soft supersymmetry breaking parameters contributing to the top squark mixing parameter, and their associated $C P$-violating phases [8]. As a result, the contribution to the final baryon asymmetry from the top squark sector turns out to be negligible. On the other hand, charginos and neutralinos may be responsible for the observed baryon asymmetry $[8,10]$. Yet, this is true within the MSSM. If the strength of the electroweak phase transition is enhanced by the presence of some new degrees of freedom beyond the ones contained in the MSSM, e.g., some extra standard model gauge singlets, light top squarks (predominantly the right-handed ones) and charginos or neutralinos are expected to give quantitatively the same contribution to the final baryon asymmetry.

While in the past few years a lot of effort has been devoted to the study of the strength of the phase transition within the MSSM, only very recently some attention has been paid to the mechanism by which the baryon asymmetry is produced. The baryon asymmetry has been usually computed using a number of steps $[7,8,17]$. (1) Look for those charges which are approximately conserved in the symmetric phase, so that they can efficiently diffuse in front of the bubble where baryon number violation is fast, and nonorthogonal to baryon number, so that the generation of a nonzero baryon charge is energetically favored. Charges with 
these characteristics in the MSSM are the axial top squark charge and the Higgsino charge, which may be produced from the interactions of squarks and charginos and/or neutralinos with the bubble wall, provided a source of $C P$ violation is present in these sectors. (2) Compute the $C P$-violating currents of the plasma locally induced by the passage of the bubble wall. (3) Write and solve a set of coupled differential diffusion equations for the local particle densities, including the $C P$-violating source terms derived from the computation of the current at step (2) and the particle number changing reactions [14]. The solution to these equations gives a net baryon number which is produced in the symmetric phase and then transmitted into the interior of the bubbles of the broken phase, where it is not wiped out if the first transition is strong enough. Notice that the $C P$-violating sources are inserted into the diffusion equations by hand only after the $C P$-violating currents have been defined and computed. This procedure introduces some degree of arbitrariness, and, indeed, different $C P$-violating sources have been adopted for the top squark and the Higgsino sectors in the literature $[7,8]$. This is not an academic question. Adopting different sources leads to different numerical results for the final baryon asymmetry, especially if the sources are expressed in terms of a different number of derivatives of the Higgs bubble wall profile and, therefore, in terms of different powers of the bubble wall velocity $v_{\omega}$ and bubble wall width $L_{\omega}$.

This crucial issue has been recently investigated in Ref. [15] where it was shown that nonequilibrium quantum field theory provides us with the necessary tools to write down a set of quantum Boltzmann equations (QBE's) describing the local particle densities and automatically incorporating the $C P$-violating sources. The most appropriate extension of the field theory to deal with these issues is to generalize the time contour of integration to a closed time path (CTP). The CTP formalism is a powerful Green's function formulation for describing nonequilibrium phenomena in field theory. It leads to a complete nonequilibrium quantum kinetic theory approach and to a rigorous computation of the $C P$-violating sources for the top squark and the Higgsino numbers. In this way, the latter have been rigorously defined and the level of arbitrariness of the previous treatments has been dismissed [15]. What is more relevant, though, is that the $C P$-violating sources-and more generally the particle number changing interactions-built up from the CTP formalism are characterized by "memory" effects which are typical of the quantum transport theory [16-18]. $C P$-violating sources are built up when right-handed top squarks and Higgsinos scatter off the advancing Higgs bubble wall and $C P$ is violated at the vertices of interactions. In the classical kinetic theory the "scattering term" does not include any integral over the past history of the system. This is equivalent to assuming that any collision in the plasma does not depend upon the previous ones. On the contrary, the quantum approach reveals that the $C P$-violating source is manifestly non-Markovian. As we shall see, these memory effects enhance the value of the final baryon asymmetry by at least two orders of magnitude with respect to the previous results. This means that the lower bound on the $C P$-violating phases from requiring successful baryogenesis is considerably relaxed-phases as large as $10^{-3}$ or smaller are enough to generate the observed baryon symmetry. This has important implications for the supersymmetric $C P$ problem. We will also investigate the structure of the kinetic QBE's derived with the CTP formalism. These equations have an obvious interpretation in terms of gain and loss processes. However, the equations are manifestly nonMarkovian and only the assumption that the relaxation time scale of the particle asymmetry is much longer than the time scale of the nonlocal kernels leads to a Markovian description. Further approximations lead to the familiar Boltzmann equations.

The paper is organized as follows. In Sec. II we give a brief description of the basic features of the nonequilibrium quantum field theory and the CTP formalism. In Secs. III and IV we compute the quantum transport equations for local particle asymmetries in the bosonic and fermion case, respectively. Section V contains the explicit computation of the $C P$-violating source for the right-handed top squark and the discussion about how to go from general QBE's to diffusion or Boltzmann equations. Section VI is devoted to the same issues, but for the Higgsino number. Section VII is devoted to the computation of the final baryon asymmetry and comparison to previous results. We conclude with an outlook of our findings in Sec. VIII.

\section{THE SCHWINGER-KELDYSH FORMALISM FOR NONEQUILIBRIUM QUANTUM FIELD THEORY}

In this section we will briefly present some of the basic features of the nonequilibrium quantum field theory based on the Schwinger-Keldysh formulation [19]. The interested reader is referred to the excellent review by Chou et al. [20] for a more exhaustive discussion.

Since we need the temporal evolution of the particle asymmetries with definite initial conditions and not simply the transition amplitude of particle reactions, the ordinary equilibrium quantum field theory at finite temperature is not the appropriate tool. The most appropriate extension of the field theory to deal with nonequilibrium phenomena amounts to generalizing the time contour of integration to a closedtime path. More precisely, the time integration contour is deformed to run from $-\infty$ to $+\infty$ and back to $-\infty$.

The CTP formalism (often dubbed as in-in formalism) is a powerful Green's function formulation for describing nonequilibrium phenomena in field theory. It allows us to describe phase-transition phenomena and to obtain a selfconsistent set of quantum Boltzmann equations. The formalism yields various quantum averages of operators evaluated in the in-state without specifying the out-state. On the contrary, the ordinary quantum field theory (often dubbed as in-out formalism) yields quantum averages of the operators evaluated with an in-state at one end and an out-state at the other.

Because of the time contour deformation, the partition function in the in-in formalism for a complex scalar field is defined to be 


$$
\begin{aligned}
Z\left[J, J^{\dagger}\right]= & \operatorname{Tr}\left(T\left\{\exp \left[i \int_{C}\left(J \boldsymbol{\phi}+J^{\dagger} \boldsymbol{\phi}^{\dagger}\right)\right]\right\} \rho\right) \\
= & \operatorname{Tr}\left(T_{+}\left\{\exp \left[i \int\left(J_{+} \boldsymbol{\phi}_{+}+J_{+}^{\dagger} \boldsymbol{\phi}_{+}^{\dagger}\right)\right]\right\}\right. \\
& \left.\times T_{-}\left\{\exp \left[-i \int\left(J_{-} \boldsymbol{\phi}_{-}+J_{-}^{\dagger} \boldsymbol{\phi}_{-}^{\dagger}\right)\right]\right\} \rho\right)
\end{aligned}
$$

where the suffice $C$ in the integral denotes that the time integration contour runs from minus infinity to plus infinity and then back to minus infinity again. The symbol $\rho$ represents the initial density matrix and the fields are in the Heisenberg picture and defined on this closed time contour. As with the Euclidean time formulation, scalar (fermionic) fields $\phi$ are still periodic (antiperiodic) in time, but with $\phi(t, \vec{x})=\phi(t-i \beta, \vec{x}), \beta=1 / T$. The temperature appears due to the boundary condition, but time is now explicitly present in the integration contour.

We must now identify field variables with arguments on the positive or negative directional branches of the time path. This doubling of field variables leads to six different realtime propagators on the contour [20]. These six propagators are not independent, but using all of them simplifies the notation. For a generic bosonic charged scalar field $\phi$ they are defined as

$$
\begin{aligned}
& G_{\phi}^{>}(x, y)=-i\left\langle\phi(x) \phi^{\dagger}(y)\right\rangle, \\
& G_{\phi}^{<}(x, y)=-i\left\langle\phi^{\dagger}(y) \phi(x)\right\rangle, \\
& G_{\phi}^{t}(x, y)=\theta(x, y) G_{\phi}^{>}(x, y)+\theta(y, x) G_{\phi}^{<}(x, y), \\
& G_{\phi}^{\bar{t}}(x, y)=\theta(y, x) G_{\phi}^{>}(x, y)+\theta(x, y) G_{\phi}^{<}(x, y), \\
& G_{\phi}^{r}(x, y)=G_{\phi}^{t}-G_{\phi}^{<}=G_{\phi}^{>}-G_{\phi}^{\bar{t}}, \\
& G_{\phi}^{a}(x, y)=G_{\phi}^{t}-G_{\phi}^{>}=G_{\phi}^{<}-G_{\phi}^{\bar{t}},
\end{aligned}
$$

where the last two Green functions are the retarded and advanced Green functions, respectively, and $\theta(x, y)=\theta\left(t_{x}\right.$ $\left.-t_{y}\right)$ is the step function. For a generic fermion field $\psi$ the six different propagators are analogously defined as

$$
\begin{aligned}
& G_{\psi}^{>}(x, y)=-i\langle\psi(x) \bar{\psi}(y)\rangle, \\
& G_{\psi}^{<}(x, y)=+i\langle\bar{\psi}(y) \psi(x)\rangle, \\
& G_{\psi}^{t}(x, y)=\theta(x, y) G_{\psi}^{>}(x, y)+\theta(y, x) G_{\psi}^{<}(x, y), \\
& G_{\psi}^{\bar{t}}(x, y)=\theta(y, x) G_{\psi}^{>}(x, y)+\theta(x, y) G_{\psi}^{<}(x, y), \\
& G_{\psi}^{r}(x, y)=G_{\psi}^{t}-G_{\psi}^{<}=G_{\psi}^{>}-G_{\psi}^{\bar{t}}, \\
& \quad G_{\psi}^{a}(x, y)=G_{\psi}^{t}-G_{\psi}^{>}=G_{\psi}^{<}-G_{\psi}^{\bar{t}} .
\end{aligned}
$$

For equilibrium phenomena, the brackets $\langle\cdots\rangle$ imply a thermodynamic average over all the possible states of the sys- tem. While for homogeneous systems in equilibrium, the Green functions depend only upon the difference of their arguments $(x, y)=(x-y)$ and there is no dependence upon $(x+y)$, for systems out of equilibrium, the definitions (2) and (3) have a different meaning. The concept of thermodynamic averaging is now ill defined. Instead, the bracket means the need to average over all the available states of the system for the nonequilibrium distributions. Furthermore, the arguments of the Green functions $(x, y)$ are not usually given as the difference $(x-y)$. For example, nonequilibrium could be caused by transients which make the Green functions depend upon $\left(t_{x}, t_{y}\right)$ rather than $\left(t_{x}-t_{y}\right)$.

For interacting systems whether in equilibrium or not, one must define and calculate self-energy functions. Again, there are six of them: $\Sigma^{t}, \Sigma^{\bar{t}}, \Sigma^{<}, \Sigma^{>}, \Sigma^{r}$, and $\Sigma^{a}$. The same relationships exist among them as for the Green functions in Eqs. (2) and (3), such as

$$
\Sigma^{r}=\Sigma^{t}-\Sigma^{<}=\Sigma^{>}-\Sigma^{\bar{t}}, \quad \Sigma^{a}=\Sigma^{t}-\Sigma^{>}=\Sigma^{<}-\Sigma^{\bar{t}} .
$$

The self-energies are incorporated into the Green functions through the use of Dyson's equations. A useful notation may be introduced which expresses four of the six Green functions as the elements of two-by-two matrices [21]

$$
\widetilde{G}=\left(\begin{array}{cc}
G^{t} & \pm G^{<} \\
G^{>} & -G^{\bar{t}}
\end{array}\right), \quad \tilde{\Sigma}=\left(\begin{array}{cc}
\Sigma^{t} & \pm \Sigma^{<} \\
\Sigma^{>} & -\Sigma^{\bar{t}}
\end{array}\right),
$$

where the upper signs refer to bosonic case and the lower signs to fermionic case. For systems either in equilibrium or nonequilibrium, Dyson's equation is most easily expressed by using the matrix notation

$$
\begin{aligned}
\widetilde{G}(x, y)= & \widetilde{G}^{0}(x, y)+\int d^{4} x_{3} \int d^{4} x_{4} \\
& \times \widetilde{G}^{0}\left(x, x_{3}\right) \tilde{\Sigma}\left(x_{3}, x_{4}\right) \widetilde{G}\left(x_{4}, y\right),
\end{aligned}
$$

where the superscript 0 on the Green functions means to use those for noninteracting systems. This equation appears quite formidable; however, some simple expressions may be obtained for the respective Green functions. It is useful to notice that Dyson's equation can be written in an alternate form, instead of Eq. (6), with $\widetilde{G}^{0}$ on the right in the interaction terms,

$$
\begin{aligned}
\widetilde{G}(x, y)= & \widetilde{G}^{0}(x, y)+\int d^{4} x_{3} \int d^{4} x_{4} \\
& \times \widetilde{G}\left(x, x_{3}\right) \tilde{\Sigma}\left(x_{3}, x_{4}\right) \widetilde{G}^{0}\left(x_{4}, y\right) .
\end{aligned}
$$

Equations (6) and (7) are the starting points to derive the quantum Boltzmann equations describing the temporal evolution of the $C P$-violating particle density asymmetries. 


\section{QBE FOR BOSONIC PARTICLE DENSITY ASYMMETRY}

From now on we will adopt the general method of deriving the QBE's provided by Kadanoff and Baym [22]. In this section our goal is to find the QBE for the generic bosonic $C P$-violating current

$$
\left\langle J_{\phi}^{\mu}(x)\right\rangle \equiv i\left\langle\phi^{\dagger}(x) \stackrel{\leftrightarrow}{\partial}_{x}^{\mu} \phi(x)\right\rangle \equiv\left[n_{\phi}(x), \vec{J}_{\phi}(x)\right] .
$$

The zero component of this current $n_{\phi}$ represents the number density of particles minus the number density of antiparticles and is therefore the quantity which enters the diffusion equations of supersymmetric electroweak baryogenesis.

Since the $C P$-violating current can be expressed in terms of the Green function $G_{\phi}^{<}(x, y)$ as

$$
\left\langle J_{\phi}^{\mu}(x)\right\rangle=-\left.\left(\partial_{x}^{\mu}-\partial_{y}^{\mu}\right) G_{\phi}^{<}(x, y)\right|_{x=y},
$$

the problem is reduced to find the QBE for the interacting Green function $G_{\phi}^{<}(x, y)$ when the system is not in equilibrium. This equation can be found from Eq. (6) by operating by $\left(\vec{\square}_{x}+m^{2}\right)$ on both sides of the equation. Here $m$ represents the bare mass term of the field $\phi$. On the right-hand side, this operator acts only on $\widetilde{G}_{\phi}^{0}$ :

$$
\begin{aligned}
& \left(\vec{\square}_{x}+m^{2}\right) \widetilde{G}_{\phi}(x, y) \\
& \quad=\delta^{(4)}(x, y) \widetilde{I}_{4}+\int d^{4} x_{3} \widetilde{\Sigma}_{\phi}\left(x, x_{3}\right) \widetilde{G}_{\phi}\left(x_{3}, y\right),
\end{aligned}
$$

where $I$ is the identity matrix. It is useful to also have an equation of motion for the other variable $y$. This is obtained from Eq. (7) by operating by $\left(\overleftarrow{\square}_{y}+m^{2}\right)$ on both sides of the equation. We obtain

$$
\begin{aligned}
& \widetilde{G}_{\phi}(x, y)\left(\overleftarrow{\square}_{y}+m^{2}\right) \\
& \quad=\delta^{(4)}(x, y) \widetilde{I}_{4}+\int d^{4} x_{3} \widetilde{G}_{\phi}\left(x, x_{3}\right) \widetilde{\Sigma}_{\phi}\left(x_{3}, y\right) .
\end{aligned}
$$

The two equations (10) and (11) are the starting point for the derivation of the QBE for the particle asymmetries. Let us extract from Eqs. (10) and (11) the equations of motions for the Green function $G_{\phi}^{<}(x, y)$

$$
\begin{aligned}
\left(\vec{\square}_{x}+m^{2}\right) G_{\phi}^{<}(x, y)= & \int d^{4} x_{3}\left[\Sigma_{\phi}^{t}\left(x, x_{3}\right) G_{\phi}^{<}\left(x_{3}, y\right)\right. \\
& \left.-\Sigma_{\phi}^{<}\left(x, x_{3}\right) G_{\phi}^{\bar{t}}\left(x_{3}, y\right)\right], \\
G_{\phi}^{<}(x, y)\left(\dot{\square}_{y}+m^{2}\right)= & \int d^{4} x_{3}\left[G_{\phi}^{t}\left(x, x_{3}\right) \Sigma_{\phi}^{<}\left(x_{3}, y\right)\right. \\
& \left.-G_{\phi}^{<}\left(x, x_{3}\right) \Sigma_{\phi}^{\bar{t}}\left(x_{3}, y\right)\right] .
\end{aligned}
$$

If we now substract the two equations and make the identification $x=y$, the left-hand side is given by

$$
\begin{aligned}
\left.\partial_{\mu}^{x}\left[\left(\partial_{x}^{\mu}-\partial_{y}^{\mu}\right) G_{\phi}^{<}(x, y)\right]\right|_{x=y} \\
=-\frac{\partial J_{\phi}^{\mu}(X)}{\partial X^{\mu}}=-\left(\frac{\partial n_{\phi}}{\partial T}+\vec{\nabla} \cdot \vec{J}_{\phi}\right),
\end{aligned}
$$

and the QBE for the particle density asymmetry is therefore obtained to be

$$
\begin{aligned}
\frac{\partial n_{\phi}(X)}{\partial T}+\vec{\nabla} \cdot \vec{j}_{\phi}(X)= & -\int d^{4} x_{3}\left[\Sigma_{\phi}^{t} G_{\phi}^{<}-\Sigma_{\phi}^{<} G_{\phi}^{\bar{t}}\right. \\
& \left.-G_{\phi}^{t} \Sigma_{\phi}^{<}-G_{\phi}^{<} \Sigma_{\phi}^{\bar{t}}\right]\left.\right|_{x=y},
\end{aligned}
$$

where we have defined the center-of-mass coordinate system

$$
X=(T, \vec{X})=\frac{1}{2}(x+y), \quad(t, \vec{r})=x-y .
$$

Notice that $T$ now means the center-of-mass time and not temperature. The identification $x=y$ in Eq. (15) is therefore equivalent to requiring $t=\vec{r}=0$.

In order to examine the "scattering term" on the righthand side of Eq. (15), the first step is to restore all the variable arguments. Setting $x=y$ in the original notation of $\Sigma_{\phi}\left(x, x_{3}\right) G_{\phi}\left(x_{3}, y\right)$ gives $\left(X, x_{3}\right)\left(x_{3}, X\right)$ for the pair of arguments

$$
\begin{aligned}
\frac{\partial n_{\phi}(X)}{\partial T} & +\vec{\nabla} \cdot \vec{j}_{\phi}(X) \\
= & -\int d^{4} x_{3}\left[\Sigma_{\phi}^{t}\left(X, x_{3}\right) G_{\phi}^{<}\left(x_{3}, X\right)\right. \\
& -\Sigma_{\phi}^{<}\left(X, x_{3}\right) G_{\phi}^{\bar{t}}\left(x_{3}, X\right)+G_{\phi}^{t}\left(X, x_{3}\right) \Sigma_{\phi}^{<}\left(x_{3}, X\right) \\
& \left.-G_{\phi}^{<}\left(X, x_{3}\right) \Sigma_{\phi}^{\bar{t}}\left(x_{3}, X\right)\right] .
\end{aligned}
$$

The next step is to employ the definitions in Eq. (2) to express the time-ordered functions $G_{\phi}^{t}, G_{\phi}^{\bar{t}}, \Sigma_{\phi}^{t}$, and $\Sigma_{\phi}^{\bar{t}}$ in terms of $G_{\phi}^{<}, G_{\phi}^{>}, \Sigma_{\phi}^{<}$, and $G_{\phi}^{>}$. Then the time integrals are separated into whether $t_{3}>T$ or $t_{3}<T$ and the right-hand side of Eq. (17) reads

$$
\begin{aligned}
= & -\int d^{4} x_{3}\left\{\theta\left(T-t_{3}\right)\left[\Sigma_{\phi}^{>} G_{\phi}^{<}+G_{\phi}^{<} \Sigma_{\phi}^{>}-\Sigma_{\phi}^{<} G_{\phi}^{>}-G_{\phi}^{>} \Sigma_{\phi}^{<}\right]\right. \\
& \left.+\theta\left(t_{3}-T\right)\left[\Sigma_{\phi}^{<} G_{\phi}^{<}+G_{\phi}^{<} \Sigma_{\phi}^{<}-\Sigma_{\phi}^{<} G_{\phi}^{<}-G_{\phi}^{<} \Sigma_{\phi}^{<}\right]\right\} .
\end{aligned}
$$

The term with $t_{3}>T$ all cancel, leaving $T>t_{3}$. Rearranging these terms gives [15]

$$
\begin{aligned}
& \frac{\partial n_{\phi}(X)}{\partial T}+\vec{\nabla} \cdot \vec{j}_{\phi}(X) \\
& =-\int d^{3} \vec{x}_{3} \int_{-\infty}^{T} d t_{3}\left[\Sigma_{\phi}^{>}\left(X, x_{3}\right) G_{\phi}^{<}\left(x_{3}, X\right)\right. \\
& \quad-G_{\phi}^{>}\left(X, x_{3}\right) \Sigma_{\phi}^{<}\left(x_{3}, X\right)+G_{\phi}^{<}\left(X, x_{3}\right) \Sigma_{\phi}^{>}\left(x_{3}, X\right) \\
& \left.\quad-\Sigma_{\phi}^{<}\left(X, x_{3}\right) G_{\phi}^{>}\left(x_{3}, X\right)\right] .
\end{aligned}
$$

This equation is the QBE for the particle density asymmetry and it can be explicitly checked that in the particular case in 
which interactions conserve the number of particles and the latter are neither created nor destroyed, the number asymmetry $n_{\phi}$ is conserved and obeys the equation of continuity $\partial n_{\phi} / \partial T+\vec{\nabla} \cdot \vec{j}_{\phi}=0$. During the production of the baryon asymmetry, however, particle asymmetries are not conserved. This occurs because the interactions themselves do not conserve the particle number asymmetries and there is some source of $C P$ violation in the system. The right-hand side of Eq. (19), through the general form of the self-energy $\Sigma_{\phi}$, contains all the information necessary to describe the temporal evolution of the particle density asymmetries: particle number changing reactions and $C P$-violating source terms, which will pop out from the corresponding selfenergy $\Sigma_{C P}$. If the interactions of the system do not violate $C P$, there will be no $C P$-violating sources and the final baryon asymmetry produced during supersymmetric baryogenesis will be vanishing.

As we shall see, the kinetic Eq. (19) has an obvious interpretation in terms of gain and loss processes. What is unusual, however, is the presence of the integral over the time: the equation is manifestly non-Markovian. Only the assumption that the relaxation time scale of the particle asymmetry is much longer than the time scale of the nonlocal kernels leads to a Markovian description. A further approximation, i.e., taking the upper limit of the time integral to $T \rightarrow \infty$, leads to the familiar Boltzmann equation. The physical interpretation of the integral over the past history of the system is straightforward: it leads to the typical "memory" effects which are observed in quantum transport theory $[16,18]$. In the classical kinetic theory the "scattering term" does not include any integral over the past history of the system which is equivalent to assume that any collision in the plasma does not depend upon the previous ones. On the contrary, quantum distributions possess strong memory effects and the thermalization rate obtained from the quantum transport theory may be substantially longer than the one obtained from the classical kinetic theory. As shown in Ref. [15], memory effects play a fundamental role in the determination of the $C P$-violating sources which fuel baryogenesis when transport properties allow the $C P$-violating charges to diffuse in front of the bubble wall separating the broken from the unbroken phase at the electroweak phase transition. Notice that so far we have not made any approximation and the computation is therefore valid for all shapes and sizes of the bubble wall expanding in the thermal bath during a firstorder electroweak phase transition.

\section{QBE FOR FERMIONIC PARTICLE DENSITY ASYMMETRY}

The generic fermionic $C P$-violating current reads

$$
\left\langle J_{\psi}^{\mu}(x)\right\rangle \equiv\left\langle\bar{\psi}(x) \gamma^{\mu} \psi(x)\right\rangle \equiv\left[n_{\psi}(x), \vec{J}_{\psi}(x)\right]
$$

where $\psi$ indicates a Dirac fermion and $\gamma^{\mu}$ represent the usual Dirac matrices. Again, the zero component of this current $n_{\psi}$ represents the number density of particles minus the number density of antiparticles and is therefore the relevant quantity for the diffusion equations of supersymmetric electroweak baryogenesis.

We want to find a couple of equations of motion for the interacting fermionic Green function $\widetilde{G}_{\psi}(x, y)$ when the system is not in equilibrium. Such equations may be found by applying the operators $\left(i \vec{b}_{x}-M\right)$ and $\left(i \hat{B}_{y}+M\right)$ on both sides of Eqs. (6) and (7), respectively. Here $M$ represents the bare mass term of the fermion $\psi$. We find

$$
\begin{aligned}
& \left(i \vec{b}_{x}-M\right) \widetilde{G}_{\psi}(x, y) \\
& \quad=\delta^{(4)}(x, y) \widetilde{I}_{4}+\int d^{4} x_{3} \widetilde{\Sigma}_{\psi}\left(x, x_{3}\right) \widetilde{G}_{\psi}\left(x_{3}, y\right), \\
& \widetilde{G}_{\psi}(x, y)\left(i \overleftarrow{b}_{y}+M\right) \\
& \quad=-\delta^{(4)}(x, y) \widetilde{I}_{4}-\int d^{4} x_{3} \widetilde{G}_{\psi}\left(x, x_{3}\right) \widetilde{\Sigma}_{\psi}\left(x_{3}, y\right) .
\end{aligned}
$$

We can now take the trace over the spinorial indices of both sides of the equations, sum up the two equations above and finally extract the equation of motion for the Green function $G_{\psi}^{>}$:

$$
\begin{aligned}
& \operatorname{Tr}\left\{\left[i \vec{b}_{x}+i \bar{b}_{y}\right] G_{\psi}^{>}(x, y)\right\} \\
& =\int d^{4} x_{3} \operatorname{Tr}\left[\Sigma_{\psi}^{>}\left(x, x_{3}\right) G_{\psi}^{t}\left(x_{3}, y\right)\right. \\
& \quad-\Sigma_{\psi}^{\bar{t}}\left(x, x_{3}\right) G_{\psi}^{>}\left(x_{3}, y\right)-G_{\psi}^{>}\left(x, x_{3}\right) \Sigma_{\psi}^{t}\left(x_{3}, y\right) \\
& \left.\quad+G_{\psi}^{\bar{t}}\left(x, x_{3}\right) \Sigma_{\psi}^{>}\left(x_{3}, y\right)\right] .
\end{aligned}
$$

Making use of the center-of-mass coordinate system, we can work out the left-hand side of Eq. (23):

$$
\begin{aligned}
\operatorname{Tr} & {\left.\left[i \vec{b}_{x} G_{\psi}^{>}(T, \vec{X}, t, \vec{r})+G_{\psi}^{>}(T, \vec{X}, t, \vec{r}) i \vec{b}_{y}\right]\right|_{t=\vec{r}=0} } \\
& =\left.i\left(\partial_{\mu}^{x}+\partial_{\mu}^{y}\right) i\left\langle\bar{\psi} \gamma^{\mu} \psi\right\rangle\right|_{t=\vec{r}=0} \\
& =-\frac{\partial}{\partial X^{\mu}}\left\langle\bar{\psi}(X) \gamma^{\mu} \psi(X)\right\rangle=-\frac{\partial}{\partial X^{\mu}} J_{\psi}^{\mu} .
\end{aligned}
$$

The next step is to employ the definitions in Eq. (3) to express the time-ordered functions $G_{\psi}^{t}, G_{\psi}^{\bar{t}}, \Sigma_{\psi}^{t}$, and $\Sigma_{\psi}^{\bar{t}}$ in terms of $G_{\psi}^{<}, G_{\psi}^{>}, \Sigma_{\psi}^{<}$, and $G_{\psi}^{>}$. The computation goes along the same lines as the analysis made in the previous section and we get [15]

$$
\begin{aligned}
\frac{\partial n_{\psi}(X)}{\partial T} & +\vec{\nabla} \cdot \vec{j}_{\psi}(X) \\
= & \int d^{3} \vec{x}_{3} \int_{-\infty}^{T} d t_{3} \operatorname{Tr}\left[\Sigma_{\psi}^{>}\left(X, x_{3}\right) G_{\psi}^{<}\left(x_{3}, X\right)\right. \\
& -G_{\psi}^{>}\left(X, x_{3}\right) \Sigma_{\psi}^{<}\left(x_{3}, X\right)+G_{\psi}^{<}\left(X, x_{3}\right) \\
& \left.\times \Sigma_{\psi}^{>}\left(x_{3}, X\right)-\Sigma_{\psi}^{<}\left(X, x_{3}\right) G_{\psi}^{>}\left(x_{3}, X\right)\right] .
\end{aligned}
$$

This is the "diffusion" equation describing the temporal 
evolution of a generic fermionic number asymmetry $n_{\psi}$. As for the bosonic case, all the information regarding particlenumber-violating interactions and $C P$-violating sources are stored in the self-energy $\Sigma_{\psi}$.

\section{THE QBE FOR THE RIGHT-HANDED TOP SQUARK NUMBER}

As we mentioned in the Introduction, a strongly first order electroweak phase transition can be achieved in the presence of a top squark lighter than the top quark $[11,12]$. In order to naturally suppress its contribution to the parameter $\Delta \rho$ and hence preserve a good agreement with the precision measurements at the CERN $e^{+} e^{-}$collider LEP, it should be mainly right handed. This can be achieved if the left-handed top squark soft supersymmetry breaking mass $\tilde{m}_{\tilde{t}_{L}}$ is much larger than $M_{Z}$. Under this assumption, only the righthanded top squarks contribute to the axial top squark charge. The right-handed top squark current $J_{\tilde{t}_{R}}^{\mu}$ associated to the right-handed top squark $\tilde{t}_{R}$ is given by

$$
J_{\tilde{t}_{R}}^{\mu}=i\left(\widetilde{t}_{R}^{*} \stackrel{\partial}{\partial}^{\mu} \widetilde{t}_{R}\right) .
$$

The self-energy of the right-handed top squark contains the information about all the sources which are responsible for changing $n_{\tilde{t}_{R}}$ in the plasma: scattering processes involving the top quark Yukawa coupling, axial top-numberviolation processes, strong sphaleron interactions, and the $C P$-violating source induced by the presence of $C P$-violating phases in the interactions of the right-handed top squark with the Higgs boson background.

Equation (19) is the QBE describing the right-handed top squark number asymmetry. Solving this equation represents an Herculean task since it is integral and nonlinear. This happens because the self-energy functions $\Sigma^{>}$and $\Sigma^{<}$are also functions of the full nonequilibrium Green functions of other degrees of freedom of the system. We can make some progress, though. Since we know that $n_{\tilde{t}_{R}}$ identically vanishes if there is no $C P$-violating source in the $\mathrm{QBE}$, i.e., in absence of the Higgs boson configuration describing the bubble wall profile, we first decompose the generic Green function $\widetilde{G}$ and self-energy function $\widetilde{\Sigma}$ as

$$
\widetilde{G}=\widetilde{G}^{0}+\delta \widetilde{G}, \quad \widetilde{\Sigma}=\widetilde{\Sigma}^{0}+\delta \tilde{\Sigma},
$$

where $\widetilde{G}^{0}$ and $\widetilde{\Sigma}^{0}$ represent the fully interacting equilibrium Green functions and self-energy functions in the unbroken phase (that is in the absence of the Higgs boson profile). This approximation amounts to retaining only the first-order linear response in the "Higgs boson insertion expansion" around the symmetric phase $\left\langle H_{i}^{0}(x)\right\rangle=v_{i}(x)=0 \quad(i=1,2)$. This is certainly a good approximation for the case under investigation. We will return to this point later.

If we now linearize Eqs. (6) and (7) and repeat the procedure described in Sec. III, we obtain

$$
\begin{aligned}
\frac{\partial n_{\tilde{t}_{R}}}{\partial T}+\vec{\nabla} \cdot \vec{j} \tilde{t}_{R}= & -\int d^{3} \vec{x}_{3} \int_{-\infty}^{T} d t_{3}\left[\delta \Sigma_{\tilde{t}_{R}}^{>} G_{\tilde{t}_{R}}^{0,<}-G_{\tilde{t}_{R}}^{0,>} \delta \Sigma_{\tilde{t}_{R}}^{<}\right. \\
& +G_{\tilde{t}_{R}}^{0,<} \delta \Sigma_{\tilde{t}_{R}}^{>}-\delta \Sigma_{\tilde{t}_{R}}^{<} G_{\tilde{t}_{R}}^{0,>}+\Sigma_{\tilde{t}_{R}}^{0,>} \delta G_{\tilde{t}_{R}}^{<} \\
& \left.-\delta G_{\tilde{t}_{R}}^{>} \Sigma_{\tilde{t}_{R}}^{0,<}+\delta G_{\tilde{t}_{R}}^{<} \sum_{\tilde{t}_{R}}^{0,>}-\Sigma_{\tilde{t}_{R}}^{0,<} \delta G_{\tilde{t}_{R}}^{>}\right],
\end{aligned}
$$

where we have used the fact that $n_{\tilde{t}_{R}}^{0}=\vec{j}_{\tilde{t}_{R}}^{0}=0$ and therefore the right-handed top squark number asymmetry and current are associated to the Green function $\delta G_{\tilde{t}_{R}}^{<}$.

In the spirit of the diagrammatic approach in the Higgs insertion expansion, we can now write the shift in the selfenergy as

$$
\delta \Sigma_{\tilde{t}_{R}}=\delta \Sigma_{\tilde{t}_{R}}^{C P}+\delta \Sigma_{\tilde{t}_{R}}^{\mathrm{int}}+\cdots
$$

where $\delta \Sigma_{\tilde{t}_{R}}^{C P}$ is the part of the self-energy responsible for the appearance of the $C P$-violating source and $\delta \Sigma_{\tilde{t}_{R}}^{\text {int }}$ accounts the interactions which change the small particle number asymmetry of the right-handed top squark originated at the passage of the Higgs boson profile through a given region of space. Here the dots represent other terms in the self-energy describing the interactions which do not change the small particle number asymmetry of the right-handed top squark (elastic scatterings) - they will not give any contribution to the right-hand side of the QBE.

Equation (28) contains in the right-hand side generic expressions such as

$$
\delta \Sigma_{\tilde{t}_{R}}^{C P} G_{\tilde{t}_{R}}^{0}+\delta \Sigma_{\tilde{t}_{R}}^{\mathrm{int}} G_{\tilde{t}_{R}}^{0}+\Sigma_{\tilde{t}_{R}}^{0} \delta G_{\tilde{t}_{R}}
$$

and

$$
G_{\tilde{t}_{R}}^{0} \delta \Sigma_{\tilde{t}_{R}}^{C P}+G_{\tilde{t}_{R}}^{0} \delta \Sigma_{\tilde{t}_{R}}^{\mathrm{int}}+\delta G_{\tilde{t}_{R}} \Sigma_{\tilde{t}_{R}}^{0} .
$$

Let us first analyze the $C P$-violating source for the righthanded top squark number induced by a novanishing $\delta \Sigma_{\tilde{t}_{R}}^{C P}$.

The CP-violating source for the right-handed top squark number. The interaction which is responsible for the $C P$-violating source right-handed top squark $\tilde{t}_{R}$, the lefthanded top squark $\tilde{t}_{L}$, and the two neutral Higgs bosons $H_{1,2}^{0}$ is given by

$$
\mathcal{L}=h_{t} \tilde{t}_{L}\left(A_{t} H_{2}^{0}-\mu^{*} H_{1}^{0}\right) \tilde{t}_{R}^{*}+\text { H.c. }
$$

Here the soft trilinear term $A_{t}$ and the supersymmetric mass term $\mu$ are meant to be complex parameters so that $\operatorname{Im}\left(A_{t} \mu\right)$ is nonvanishing.

At the lowest level of perturbation, the interactions (32) induce a contribution to the self-energy of the form [15]

$$
\begin{aligned}
& \delta \Sigma_{\tilde{t}_{R}}^{C P,>}(x, y)=g^{C P}(x, y) G_{\tilde{t}_{L}}^{>}(x, y), \\
& \delta \Sigma^{C P,<}(x, y)=g^{C P}(x, y) G_{\tilde{t}_{L}}^{<}(x, y) .
\end{aligned}
$$


Here

$$
g_{C P}(x, y)=h_{t}^{2}\left[A_{t}^{*} v_{2}(x)-\mu v_{1}(x)\right]\left[A_{t} v_{2}(y)-\mu^{*} v_{1}(y)\right]
$$

and we can safely approximate the exact left-handed top squark Green functions $G_{\tilde{t}_{L}}$ with the corresponding fully interacting equilibrium Green functions in the unbroken phase $G_{\tilde{t}_{L}}^{0}$. This is because any departure from thermal equilibrium distribution functions is caused at a given point by the passage of the wall and, therefore, is $\mathcal{O}\left(v_{\omega}\right)$. Since we will show that the source is already linear in $v_{\omega}$, working with thermal equilibrium Green functions in the unbroken phase amounts to ignoring terms of higher order in $v_{\omega}$. This is accurate as the bubble wall is moving slowly in the plasma.

If we now insert the expressions (33) and (34) into the QBE (28), we get the right-handed top squark $C P$-violating source [15]

$$
\begin{aligned}
\mathcal{S}_{\tilde{t}_{R}}= & -2 i \int d^{3} \vec{x}_{3} \int_{-\infty}^{T} d t_{3}\left[g_{C P}\left(X, x_{3}\right)-g_{C P}\left(x_{3}, X\right)\right] \\
& \times \operatorname{Im}\left[G_{\tilde{t}_{L}}^{0,>}\left(X, x_{3}\right) G_{\tilde{t}_{R}}^{0,<}\left(x_{3}, X\right)\right]+\cdots \\
= & 4 h_{t}^{2} \int d^{3} \vec{x}_{3} \int_{-\infty}^{T} d t_{3} \operatorname{Im}\left(A_{t} \mu\right) \\
& \times\left[v_{2}(X) v_{1}\left(x_{3}\right)-v_{2}\left(x_{3}\right) v_{1}(X)\right] \\
& \times \operatorname{Im}\left[G_{\tilde{t}_{L}}^{0,>}\left(X, x_{3}\right) G_{\tilde{t}_{R}}^{0,<}\left(x_{3}, X\right)\right] .
\end{aligned}
$$

$\mathcal{S}_{\tilde{t}_{R}}$ vanishes if the relative phase of $A_{t} \mu$ is zero and if the ratio $\tan \beta(x) \equiv v_{2}(x) / v_{1}(x)$ is a constant in the interior of the bubble wall.

Notice that the source is built up integrating over all the history of the system. It is exactly this "memory effect" that is responsible for the enhancement of the final baryon asymmetry. Furthermore, the source is constructed when the righthanded top squarks pass across the wall, they first scatter off the wall and are transformed into left-handed top squarks; the latter subsequently suffer another scattering off the wall and are converted again into right-handed top squarks. If $C P$ violation is taking place in both interactions, a nonvanishing $C P$-violating source $\mathcal{S}_{\tilde{t}_{R}}$ pops out from the thermal bath.

In order to deal with analytic expressions, we can work out the thick wall limit and simplify the expressions obtained above by performing a derivative expansion

$$
v_{i}\left(x_{3}\right)=\sum_{n=0}^{\infty} \frac{1}{n !} \frac{\partial^{n}}{\partial\left(X^{\mu}\right)^{n}} v_{i}(X)\left(x_{3}^{\mu}-X^{\mu}\right)^{n} .
$$

The term with no derivatives vanishes in the expansion (36), $v_{2}(X) v_{1}(X)-v_{1}(X) v_{2}(X)=0$, which means that the static term in the derivative expansion does not contribute to the source $\mathcal{S}_{\tilde{t}_{R}}$. For a smooth Higgs boson profile, the derivatives with respect to the time coordinate and $n>1$ are associated with higher powers of $v_{\omega} / L_{\omega}$, where $v_{\omega}$ and $L_{\omega}$ are the velocity and the width of the bubble wall, respectively. Since the typical time scale of the processes giving rise to the source is given by the thermalization time of the top squarks $1 / \Gamma_{\tilde{t}}$, the approximation is good for values of $L_{\omega} \Gamma_{\tilde{t}} / v_{\omega}$ $\gg 1$. In other words, this expansion is valid only when the mean free path of the top squarks in the plasma is smaller than the scale of variation of the Higgs boson background determined by the wall thickness $L_{\omega}$ and the wall velocity $v_{\omega}$. A detailed computation of the thermalization rate of the right-handed top squark from the imaginary part of the twopoint Green function has been recently performed in Ref. [23] by making use of improved propagators and including resummation of hard thermal loops. ${ }^{1}$ The thermalization rate has been computed exactly at the one-loop level in the hightemperature approximation as a function of the plasma righthanded top squark mass $m_{\tilde{t}_{R}}(T)$ and an estimate for the magnitude of the two-loop contributions which dominate the rate for small $m_{\tilde{t}_{R}}(T)$ was also given. If $m_{\tilde{t}_{R}}(T) \geq T$, the thermalization is dictated by the one-loop thermal decay rate which can be larger than $T[23] .^{2}$

The term corresponding to $n=1$ in the expansion (36) gives a contribution to the source proportional to the function

$$
v_{1}(X) \partial_{X}^{\mu} v_{2}(X)-v_{2}(X) \partial_{X}^{\mu} v_{1}(X) \equiv v^{2}(X) \partial_{X}^{\mu} \beta(X),
$$

which should vanish smoothly for values of $X$ outside the bubble wall. Here we have denoted $v^{2} \equiv v_{1}^{2}+v_{2}^{2}$. Since the variation of the Higgs fields is due to the expansion of the bubble wall through the thermal bath, the source $\mathcal{S}_{\tilde{t}_{R}}$ will be linear in $v_{\omega}$. The corresponding contribution to the $C P$-violating source reads [15]

$$
\mathcal{S}_{\tilde{t}_{R}}(X)=h_{t}^{2} \operatorname{Im}\left(A_{t} \mu\right) v^{2}(X) \dot{\beta}(X) \mathcal{I}_{\tilde{t}_{R}}
$$

where $\dot{\beta}(X) \equiv d \beta(X) / d t_{X}$,

$$
\begin{aligned}
\mathcal{I}_{\tilde{t}_{R}}= & \int_{0}^{\infty} d k \frac{k^{2}}{2 \pi^{2} \omega_{\tilde{t}_{L}} \omega_{\tilde{t}_{R}}} \\
& \times\left\{\left[1+2 \operatorname{Re}\left(f_{\tilde{t}_{L}}^{0}\right)\right] I\left(\omega_{\tilde{t}_{R}}, \Gamma_{\tilde{t}_{R}}, \omega_{\tilde{t}_{L}}, \Gamma_{\tilde{t}_{L}}\right)\right. \\
& +\left[1+2 \operatorname{Re}\left(f_{\tilde{t}_{R}}^{0}\right)\right] I\left(\omega_{\tilde{t}_{L}}, \Gamma_{\tilde{t}_{L}}, \omega_{\tilde{t}_{R}}, \Gamma_{\tilde{t}_{R}}\right) \\
& \left.-2\left[\operatorname{Im}\left(f_{\tilde{t}_{R}}^{0}\right)+\operatorname{Im}\left(f_{\tilde{t}_{L}}^{0}\right)\right] G\left(\omega_{\tilde{t}_{R}}, \Gamma_{\tilde{t}_{R}}, \omega_{\tilde{t}_{L}}, \Gamma_{\tilde{t}_{L}}\right)\right\}
\end{aligned}
$$

\footnotetext{
${ }^{1}$ The left-handed top squark is usually considered to be much heavier than $T$ and its decay width corresponds to the one in the present vacuum.

${ }^{2}$ For smaller values of $m_{\tilde{t}_{R}}(T)$, when the thermalization is dominated by two-loop effects (i.e., scattering), $\Gamma_{\tilde{t}_{R}}$ may be as large as $10^{-3} T[23]$.
} 
and $f_{\tilde{t}_{R}(L)}^{0}=1 /\left[\exp \left(\omega_{\tilde{t}_{R}(L)} / T+i \Gamma_{\tilde{t}_{R}(L)} / T\right)-1\right]$ are the equilibrium distribution functions in the unbroken phase. ${ }^{3}$

The functions $I$ and $G$ are given by

$$
\begin{aligned}
I(a, b, c, d)= & \frac{1}{2} \frac{1}{\left[(a+c)^{2}+(b+d)^{2}\right]} \sin \left[2 \arctan \frac{a+c}{b+d}\right] \\
& +\frac{1}{2} \frac{1}{\left[(a-c)^{2}+(b+d)^{2}\right]} \sin \left[2 \arctan \frac{a-c}{b+d}\right], \\
G(a, b, c, d)= & -\frac{1}{2} \frac{1}{\left[(a+c)^{2}+(b+d)^{2}\right]} \cos \left[2 \arctan \frac{a+c}{b+d}\right] \\
& -\frac{1}{2} \frac{1}{\left[(a-c)^{2}+(b+d)^{2}\right]} \cos \left[2 \arctan \frac{a-c}{b+d}\right] .
\end{aligned}
$$

Notice that the function $G\left(\omega_{\tilde{t}_{R}}, \Gamma_{\tilde{t}_{R}}, \omega_{\tilde{t}_{L}}, \Gamma_{\tilde{t}_{L}}\right)$ has a peak for $\omega_{\tilde{t}_{R}} \sim \omega_{\tilde{t}_{L}}$. This resonant behavior $[8,15]$ is associated to the fact that the Higgs background is carrying a very low momentum (of order of the inverse of the bubble wall width $L_{\omega}$ ) and to the possibility of absorption or emission of Higgs boson quanta by the propagating supersymmetric particles. The resonance can only take place when the left-handed top squark and the right-handed top squark do not differ too much in mass. By using the uncertainty principle, it is easy to understand that the width of this resonance is expected to be proportional to the thermalization rate of the particles giving rise to the baryon asymmetry. Within the MSSM, however, it is assumed that the left-handed top squark mass $m_{\tilde{t}_{L}}$ is much larger than the temperature $T$ and the resonance can only happen for momenta larger than $m_{\tilde{t}_{L}}$. Such configurations are exponentially suppressed and do not give any relevant contribution to the $C P$-violating source. Nonetheless, if the electroweak phase transition is enhanced by the presence of some new degrees of freedom beyond the ones contained in the MSSM, e.g., some extra standard model gauge singlets, the resonance effects in the top squark sector might be relevant.

The inelastic interactions of the right-handed top squark number. Let us now investigate the other terms in Eqs. (30) and (31) leading to particle number changing interactions. The inelastic scattering processes which change the righthanded top squark number are the ones induced by the top Yukawa coupling and by the axial top number violation inside the Higgs bubble wall (besides the the ones arising from strong sphaleron transitions). For the sake of simplicity, let us focus on the top Yukawa interactions-other interactions may be treated similarly. If we assume that the left-handed

\footnotetext{
${ }^{3}$ To account for interactions with the surrounding plasma in the unbroken phase, particles must be substituted by quasiparticles and dressed propagators are to be adopted. This means that self-energy corrections are one and twp-loops to the particle propagators modify the dispersion relations, which becomes $\omega^{2}=\vec{k}^{2}+m^{2}(T)$ with $m(T)$ the plasma mass, and introduce a finite width $\Gamma$.
}

top squark is heavier than the temperature of the plasma and is therefore decoupled from the thermal bath, these interactions read

$$
\mathcal{L}=h_{t} \overline{\widetilde{H}_{2}^{0}} P_{L} t \tilde{t}_{R}^{*}+\text { H.c. }
$$

where $P_{L}$ denotes the left-handed chirality projector operator and $\widetilde{H}_{2}^{0}$ and $t$ are the Higgsino and top quark fields, respectively.

The right-hand side of Eq. (28) now contains generic expressions such as

$$
\delta \Sigma_{\tilde{t}_{R}}^{\mathrm{int}} G_{\tilde{t}_{R}}^{0}+\Sigma_{\tilde{t}_{R}}^{0} \delta G_{\tilde{t}_{R}}-G_{\tilde{t}_{R}}^{0} \delta \Sigma_{\tilde{t}_{R}}^{\text {int }}-\delta G_{\tilde{t}_{R}} \Sigma_{\tilde{t}_{R}}^{0} .
$$

Since the contribution to $\Sigma_{\tilde{t}_{R}}^{\text {int }}$ induced by the top Yukawa quark coupling is symbolically given by $h_{t}^{2} G_{\tilde{H}_{2}^{0} G_{t}}$, the expression (42) may be written as

$$
\begin{aligned}
\delta G_{\tilde{H}_{2}^{0}} G_{t_{L}}^{0} G_{\tilde{t}_{R}}^{0}+G_{\tilde{H}_{2}^{0}}^{0} \delta G_{t_{L}} G_{\tilde{t}_{R}}^{0}+G_{\tilde{H}_{2}^{0}}^{0} G_{t_{L}}^{0} \delta G_{\tilde{t}_{R}} \\
-G_{\tilde{t}_{R}}^{0} \delta G_{\tilde{H}_{2}^{0}} G_{t_{L}}^{0}-G_{\tilde{t}_{R}}^{0} G_{\widetilde{H}_{2}^{0}}^{0} \delta G_{t_{L}}-\delta G_{\tilde{t}_{R}} G_{\tilde{H}_{2}^{0}}^{0} G_{t_{L}}^{0} .
\end{aligned}
$$

We now show that-under a number of reasonable approximations - these terms all together lead to the familiar "scattering term" proportional to $\left(n_{\tilde{t}_{R}}-n_{t_{L}}+n_{\tilde{H}_{2}^{0}}\right)$ in the right-hand side of the diffusion equation for the right-handed top squark number.

Getting the diffusion equation. To proceed further we need to introduce reasonable approximations based on physical considerations. The main phenomena occurring in the plasma when the Higgs boson bubble wall is passing through a certain region are characterized by three different time (or length) scales: the quantum (microscopic) time $\tau_{Q}$, the statistical (macroscopic) time $\tau_{S}$, and the time set by the bubble wall thickness and velocity $\tau_{\omega} \sim L_{\omega} / v_{\omega}$. The first measures the range of radiative corrections to the Compton wavelength of particles and the second measures the range of interactions among particles. In the more familiar classic kinetic interaction theory, a similar distinction is made between the scattering length and the mean free path. It is a reasonable assumption that the statistical scale $\tau_{S}$ is much larger than the quantum one. Furthermore, in the thick wall limit, the following hierarchy holds: $\tau_{\omega} \gg \tau_{S} \gg \tau_{Q}$. This observation allows us to recast the quantum field theoretical problem into a much simpler kinetic theory and, under some further assumptions, to rederive the classical diffusion equationsusing well-known techniques from relativistic many-body theory [16]. Suppose we separate the space-time "cells" whose characteristic length scale is intermediate between the statistical and the quantum scales. As the correlation between cells will be negligible by construction, the only interesting case is when the two arguments of a given propagator or self-energy lie in the same cell. In the interior of a single cell, relaxation phenomena are negligible. More concretely, the propagators may be Fourier transformed over a cell. Relaxation phenomena become apparent as we move from cell to cell. This picture breaks down if, for instance, the major 
contribution to $C P$-violating sources come from long wavelength particles for which the mean free path becomes comparable to the Compton wavelength. We will return to this point later.

In mathematical language, if $x$ and $y$ are the arguments of a propagator or self-energy, we Fourier transform with respect to the $r=x-y$, while the absolute coordinate $X=\frac{1}{2}(x$ $+y$ ) serves as a cell label. The Fourier transform reads

$$
\begin{aligned}
G(x, y) & =\int \frac{d^{4} p}{(2 \pi)^{4}} e^{-i p \cdot(x-y)} G\left(\frac{x+y}{2}, p\right) \\
& =\int \frac{d^{4} p}{(2 \pi)^{4}} e^{-i p \cdot r} G(X, p),
\end{aligned}
$$

where $G(X, p)$ is called the Wigner transform of $G(x, y)$. Similar formulas hold for the self-energies.

Let us now have a closest look at the third term in Eq. (43). We can rewrite it as

$$
\begin{aligned}
& \sum_{\tilde{t}_{R}}^{0}\left(X, x_{3}\right) \delta G_{\tilde{t}_{R}}\left(x_{3}, X\right) \\
& =\int \frac{d^{3} \vec{q}}{(2 \pi)^{3}} e^{i \vec{q} \cdot\left(\vec{X}-\vec{x}_{3}\right)} \\
& \quad \times \int \frac{d^{3} \vec{k}}{(2 \pi)^{3}} e^{i \vec{k} \cdot\left(\vec{x}_{3}-\vec{X}\right)} \sum_{\tilde{t}_{R}}^{0}\left(\frac{\vec{X}+\vec{x}_{3}}{2}, T, t_{3}, \vec{q}\right) \\
& \quad \times \delta G_{\tilde{t}_{R}}\left(\frac{\vec{X}+\vec{x}_{3}}{2}, T, t_{3}, \vec{k}\right) .
\end{aligned}
$$

The integrand will be appreciably different from zero only when $X$ belongs to the same cell as $x_{3}$, that is $\left(X+x_{3}\right) /$ $2 \sim X$. Inserting the above expression into Eq. (28), we get

$$
-\int_{-\infty}^{T} d t_{3} \int \frac{d^{3} \vec{k}}{(2 \pi)^{3}} \Sigma_{\tilde{t}_{R}}^{0}\left(X, \vec{q}, T-t_{3}\right) \delta G_{\tilde{t}_{R}}\left(X, \vec{k}, t_{3}-T\right) .
$$

A similar expression is obtained for the last term in Eq. (43) $\delta G_{\tilde{t}_{R}} \Sigma_{\tilde{t}_{R}}^{0}$.

Let us now adopt the quasiparticle ansatz for the shift in the right-handed top squark Green function

$$
\begin{aligned}
\delta G_{\tilde{t}_{R}}^{>}(X, k)= & \frac{i \pi}{\omega_{\tilde{t}_{R}}}\left\{\left[1+\delta f_{\tilde{t}_{R}}(X, \vec{k})\right] \delta\left(k^{0}-\omega_{\tilde{t}_{R}}\right)\right. \\
& \left.+\delta \bar{f}_{\tilde{t}_{R}}(X,-\vec{k}) \delta\left(k^{0}+\omega_{\tilde{t}_{R}}\right)\right\}, \\
\delta G_{\tilde{t}_{R}}^{<}(X, k)= & \frac{i \pi}{\omega_{\tilde{t}_{R}}}\left\{\delta f_{\tilde{t}_{R}}(X, \vec{k}) \delta\left(k^{0}-\omega_{\tilde{t}_{R}}\right)\right. \\
& \left.+\left[1+\delta \bar{f}_{\tilde{t}_{R}}(X,-\vec{k})\right] \delta\left(k^{0}+\omega_{\tilde{t}_{R}}\right)\right\},
\end{aligned}
$$

where $\omega_{\tilde{t}_{R}}^{2}=\vec{k}^{2}+m_{\tilde{t}_{R}}^{2}(X, T)$ and the plasma mass squared $m_{\tilde{t}_{R}}^{2}(X, T)$ is a function of the spacetime coordinate $X$ as well as of the temperature $T$. This ansatz is the logical generalization of the Green function for free fields and incorporate the renormalization effects of the nontrivial spectral density in the plasma. In this sense the Wigner functions $\delta f_{\tilde{t}_{R}}$ and $\delta \bar{f}_{\tilde{t}_{R}}$ are the quantum kinetic extension of the classical particles phase-space distributions for the right-handed top squark and its antistate. Notice, though, that this ansatz does not solve the QBE unless one neglects off-shell terms [24]. This procedure is, however, justified if $\tau_{\omega} \gg \tau_{S} \gg \tau_{Q}$.

From this ansatz we can construct the equation for the right-handed top squark number asymmetry $n_{\tilde{t}_{R}}$ by inserting Eq. (47) into the right-hand side of Eq. (28). After some algebra and exploiting the symmetry properties of the Green functions, we find that the third and last terms of Eq. (43) lead to

$$
\begin{aligned}
\frac{\partial n_{\tilde{t}_{R}}}{\partial T}+\vec{\nabla} \cdot \vec{j}_{\tilde{t}_{R}} \propto- & \int_{-\infty}^{T} d t_{3} \int d \omega \int \frac{d^{3} \vec{k}}{(2 \pi)^{3}} \Gamma_{\tilde{t}_{R}}(\omega, \vec{k}) \\
& \times \cos \left[\left(\omega-\omega_{\tilde{t}_{R}}\right)\left(T-t_{3}\right)\right]\left(\delta f_{\tilde{t}_{R}}-\delta \bar{f}_{\tilde{t}_{R}}\right)\left(\vec{k}, t_{3}\right),
\end{aligned}
$$

where we have made use of the fact that the equilibrium self-energy $\Sigma^{0}$ does not depend on the "cell" label $X$ and

$$
\Gamma_{\tilde{t}_{R}}(\omega, \vec{k})=\frac{\sum_{\tilde{t}_{R}}^{0,>>}(\omega, \vec{k})-\Sigma_{\tilde{t}_{R}}^{0,<}(\omega, \vec{k})}{i \omega_{\tilde{t}_{R}}} .
$$

The kinetic Eq. (48) has an obvious interpretation in terms of gain and loss processes which change the number density asymmetry of the right-handed top squark. This is because the rate of change of the particle number asymmetry in the plasma is related to the imaginary part of the retarded selfenergy function, Eq. (49). However, the kinetic equation is non-Markovian and the retarded time integral and the cosine function replace the more familiar energy conserving function present in the classical kinetic equation. Under which conditions can we obtain the more familiar classical Boltzmann equation? Let us suppose first that the relaxation time scale for $n_{\tilde{t}_{R}}$ is much longer than the time scale of the nonlocal kernel. Under this assumption, $\delta f_{\tilde{t}_{R}}\left(t_{3}\right)-\delta \bar{f}_{\tilde{t}_{R}}\left(t_{3}\right)$ can be replaced by $\delta f_{\tilde{t}_{R}}(T)-\delta \bar{f}_{\tilde{t}_{R}}(T)$ and taken outside the time integral. This leads to a Markovian description. A further approximation is obtained taking the upper limit of the time integral to $T \rightarrow \infty$. The cosine function becomes an energy conserving delta function and we obtain

$$
\frac{\partial n_{\tilde{t}_{R}}}{\partial T}+\vec{\nabla} \cdot \vec{j}_{\tilde{t}_{R}} \propto-\int \frac{d^{3} \vec{k}}{(2 \pi)^{3}} \Gamma_{\tilde{t}_{R}}^{\infty}\left(\omega_{\tilde{t}_{R}}, \vec{k}\right)\left(\delta \tilde{f}_{\tilde{t}_{R}}-\delta \bar{f}_{\tilde{t}_{R}}\right)(\vec{k}, T) .
$$

This result is the familiar relationship between the relaxation time of the particle distribution $\Gamma_{\tilde{t}_{R}}^{\infty}$ and the damping rate, which is determined by the contribution to imaginary part of the self-energy on shell from the top Yukawa coupling $\Gamma_{\tilde{t}_{R}}$ $=-i\left(\Sigma_{\tilde{t}_{R}}^{0,>}-\Sigma_{\tilde{t}_{R}}^{0,<}\right) / \omega_{\tilde{t}_{R}}$. 
We can repeat the same procedure for all the other terms present in the expression (43). Making the further approximation that the relaxation rate induced by the top Yukawa coupling is the same for the left-handed top squark, the righthanded top squark, and the higgsino ${ }^{4} \Gamma_{\text {top }}$, and that, in the high-temperature limit, it depends on the three-momentum only weakly, we can take it outside of the momentum integral. Moreover, if we follow the spirit of the usual derivation of Fick's law, we end up with the familiar diffusion equation for the right-handed top squark number asymmetry

$$
\frac{\partial n_{\tilde{t}_{R}}}{\partial T}-D_{\tilde{t}_{R}} \nabla^{2} n_{\tilde{t}_{R}}=-\Gamma_{\text {top }}\left(n_{\tilde{t}_{R}}-n_{t_{L}}+n_{\tilde{H}_{2}^{0}}\right)+\mathcal{S}_{\tilde{t}_{R}}+\cdots,
$$

where

$$
n_{\tilde{t}_{R}}=\int \frac{d^{3} \vec{k}}{(2 \pi)^{3}}\left(\delta \tilde{t}_{\tilde{t}_{R}}-\delta \bar{f}_{\tilde{t}_{R}}\right)
$$

$D_{\tilde{t}_{R}}$ is the right-handed top squark diffusion coefficient and ellipses mean that one should also include the axial topnumber-violation processes as well as the strong sphaleron interactions.

To assess whether the Markovian and the Boltzmann approximations are reliable in the framework of supersymmetric electroweak baryogenesis one has to understand the different time scales. The time scale of the kernel is of the order of $\omega_{\tilde{t}_{R}}^{-1}$. Since $\Gamma_{\tilde{t}_{R}}^{-} \ll \omega_{\tilde{t}_{R}}$, the relaxation time scale of the right-handed top squark population is longer than the range of the kernel and the Markovian approximation is warranted. Furthermore, since the time scale set by the bubble wall profile $\sim L_{\omega} / v_{\omega}$ and the diffusion time scale $\sim D_{\tilde{t}_{R}} / v_{\omega}^{2}$ are larger than the relaxation time, the Boltzmann-diffusion equation is also warranted..$^{5}$

\section{THE QBE FOR THE HIGGSINO NUMBER}

The Higgs fermion current associated with neutral and charged Higgsinos can be written as

$$
J_{\widetilde{H}}^{\mu}=\overline{\widetilde{H}} \gamma^{\mu} \widetilde{H},
$$

where $\widetilde{H}$ is the Dirac spinor

\footnotetext{
${ }^{4}$ This approximation may turn out to be very rough for some choice of the parameters because the imaginary part of the twopoint self-energy of different particles depend sensitively on their dispersion relations in the plasma [23,27] and, especially for Majorana fermions, the latter are highly nontrivial [26].

${ }^{5}$ On the other hand, for soft scales and for values of the righthanded SUSY breaking mass term such that the plasma mass $m_{\tilde{t}_{R}}$ is small compared to the temperature, the relaxation time scale becomes comparable to the time scale of the kernel and the Markovian approximation breaks down.
}

$$
\widetilde{H}=\left(\begin{array}{c}
\widetilde{H}_{2} \\
\widetilde{H}_{1}
\end{array}\right)
$$

and $\widetilde{H}_{2}=\widetilde{H}_{2}^{0}\left(\widetilde{H}_{2}^{+}\right), \widetilde{H}_{1}=\widetilde{H}_{1}^{0}\left(\widetilde{H}_{1}^{-}\right)$for neutral (charged) Higgsinos. The processes in the plasma which change the Higgsino number are the ones induced by the top Yukawa coupling and by interactions with the Higgs profile. The interactions among the charginos and the charged Higgsinos which are responsible for the $C P$-violating source in the diffusion equation for the Higgs fermion number read

$$
\mathcal{L}=-g_{2}\left\{\tilde{\tilde{H}}\left[v_{1}(x) P_{L}+e^{i \theta_{\mu}} v_{2}(x) P_{R}\right] \tilde{W}\right\}+\text { H.c. },
$$

where $\theta_{\mu}$ is the phase of the $\mu$ parameter. Analogously, the interactions among the $B$-ino, the $\widetilde{W}_{3}$-ino, and the neutral Higgsinos are

$$
\begin{aligned}
\mathcal{L}= & -\frac{1}{2}\left\{\overline{\widetilde{H}}^{0}\left[v_{1}(x) P_{L}+e^{i \theta} v_{2}(x) P_{R}\right]\right. \\
& \left.\times\left(g_{2} \widetilde{W}_{3}-g_{1} \widetilde{B}\right)\right\}+ \text { H.c. }
\end{aligned}
$$

We can compute the QBE for the Higgsino number repeating step by step the procedure adopted in the previous section. To compute the source for the Higgs fermion number $\mathcal{S}_{\widetilde{H}}$ we again perform a "Higgs boson insertion expansion" around the symmetric phase. At the lowest level of perturbation, the interactions of the charged Higgsino induce a contribution to the self-energy of the form (and analogously for the other component $\delta \Sigma_{\widetilde{H}}^{C P,>}$ )

$$
\begin{aligned}
\delta \Sigma_{\tilde{H}}^{C P,<}(x, y)= & g_{C P}^{L}(x, y) P_{L} G_{\tilde{W}}^{0,<}(x, y) P_{L} \\
& +g_{C P}^{R}(x, y) P_{R} G_{\widetilde{W}}^{0,<}(x, y) P_{R},
\end{aligned}
$$

where

$$
\begin{aligned}
& g_{\mathrm{CP}}^{L}(x, y)=g_{2}^{2} v_{1}(x) v_{2}(y) e^{-i \theta_{\mu}}, \\
& g_{\mathrm{CP}}^{R}(x, y)=g_{2}^{2} v_{1}(y) v_{2}(x) e^{i \theta_{\mu} .}
\end{aligned}
$$

Again, we have approximated the exact Green function of $W$-inos $G_{\tilde{W}}$ by the equilibrium Green function in the unbroken phase $G_{\tilde{W}}^{0}$. Similar formulas hold for the neutral Higgsinos.

Analogously to the case of right-handed top squarks, the dispersion relations of charginos and neutralinos are changed by high-temperature corrections [25]. Even though fermionic dispersion relations are highly nontrivial, especially when dealing with Majorana fermions [26], relatively simple expressions for the equilibrium fermionic spectral functions may be given in the limit in which the damping rate is smaller than the typical self-energy of the fermionic excitation [18]. The computation of the source goes along the same lines of the calculation done in the previous section and it is easy to show that the $C P$-violating source [15] 


$$
\begin{aligned}
\mathcal{S}_{\widetilde{H}}= & -\int d^{3} \vec{x}_{3} \int_{-\infty}^{T} d t_{3} \operatorname{Tr}\left[\delta \Sigma_{\widetilde{H}}^{C P,>}\left(X, x_{3}\right) G_{\widetilde{H}}^{0,<}\left(x_{3}, X\right)\right. \\
& -G_{\widetilde{H}}^{0,>}\left(X, x_{3}\right) \delta \Sigma_{\widetilde{H}}^{C P,<}{ }_{\left(x_{3}, X\right)} \\
& +G_{\widetilde{H}}^{0,<}\left(X, x_{3}\right) \delta \Sigma_{\widetilde{H}}^{C P,>}\left(x_{3}, X\right) \\
& \left.-\delta \Sigma_{\widetilde{H}}^{C P,<}\left(X, x_{3}\right) G_{\widetilde{H}}^{0,>}\left(x_{3}, X\right)\right],
\end{aligned}
$$

contains in the integrand the following function:

$$
\begin{gathered}
g_{C P}^{L}\left(X, x_{3}\right)+g_{C P}^{R}\left(X, x_{3}\right)-g_{C P}^{L}\left(x_{3}, X\right)-g_{C P}^{R}\left(x_{3}, X\right) \\
=2 i \sin \theta_{\mu}\left[v_{2}(X) v_{1}\left(x_{3}\right)-v_{1}(X) v_{2}\left(x_{3}\right)\right],
\end{gathered}
$$

which vanishes if $\operatorname{Im}(\mu)=0$ and if the $\tan \beta(x)$ is a constant along the Higgs boson profile. Performing the "Higgs boson derivative expansion,', we finally get [15]

$$
\mathcal{S}_{\widetilde{H}}(X)=\operatorname{Im}(\mu)\left[v^{2}(X) \dot{\beta}(X)\right]\left[3 M_{2} g_{2}^{2} \mathcal{I}_{\widetilde{H}}^{\widetilde{W}}+M_{1} g_{1}^{2} \mathcal{I}_{\widetilde{H}}^{\widetilde{B}}\right]
$$

where

$$
\begin{aligned}
\mathcal{I}_{\widetilde{H}}^{\tilde{W}}= & \int_{0}^{\infty} d k \frac{k^{2}}{2 \pi^{2} \omega_{\tilde{H}} \omega_{\tilde{W}}}\left\{\left[1-2 \operatorname{Re}\left(f_{\tilde{W}}^{0}\right)\right] I\left(\omega_{\tilde{H}}, \Gamma_{\tilde{H}}, \omega_{\tilde{W}}, \Gamma_{\tilde{W}}\right)\right. \\
& +\left[1-2 \operatorname{Re}\left(f_{\tilde{H}}^{0}\right)\right] I\left(\omega_{\tilde{W}}, \Gamma_{\tilde{W}}, \omega_{\tilde{H}}, \Gamma_{\tilde{H}}\right) \\
& \left.+2\left[\operatorname{Im}\left(f_{\tilde{H}}^{0}\right)+\operatorname{Im}\left(f_{\tilde{W}}^{0}\right)\right] G\left(\omega_{\tilde{H}}, \Gamma_{\tilde{H}}, \omega_{\tilde{W}}, \Gamma_{\tilde{W}}\right)\right\}
\end{aligned}
$$

and $\quad \omega_{\tilde{H}(\tilde{W})}^{2}=k^{2}+|\mu|^{2}\left(M_{2}^{2}\right)$ while $f_{\tilde{H}(\tilde{W})}^{0}=1 /\left[\exp \left(\omega_{\tilde{H}(\tilde{W})} / T\right.\right.$ $\left.\left.+i \Gamma_{\tilde{H}(\tilde{W})} / T\right)+1\right]$. The exact computation of the damping rate of charged and neutral Higgsinos $\Gamma_{\tilde{H}}$ will be given elsewhere [27], but it is expected to be of the order of $5 \times 10^{-2} T$. The $B$-ino contribution may be obtained from the above expressions by replacing $M_{2}$ by $M_{1}$. As for $\mathcal{S}_{\tilde{t}_{R}}$, the $C P$-violating source for the Higgs fermion number is enhanced if $M_{2}, M_{1} \sim \mu$, and low momentum particles are transmitted over the distance $L_{\omega}$. This means that the classical approximation is not entirely adequate to describe the quantum interference nature of $C P$ violation and only a quantum approach is suitable for the computation of the building up of the $C P$-violating sources [15].

To find the "collision terms" which change the Higgsino number inside the Higgs boson bubble wall one has to repeat the analysis performed previously for the right-handed top squark number. In particular, one has to adopt the quasiparticle ansatz for the Higgsinos. The one for charged Higgsinos, for instance, reads

$$
\begin{aligned}
\delta G_{\tilde{H}}^{>}(X, k)= & -\frac{i \pi}{\omega_{\tilde{H}}}(k+|\mu|)\left\{\left[1+\delta f_{\tilde{H}}(X, \vec{k})\right] \delta\left(k^{0}-\omega_{\tilde{H}}\right)\right. \\
& \left.+\delta \bar{f}_{\tilde{H}}(X,-\vec{k}) \delta\left(k^{0}+\omega_{\tilde{H}}\right)\right\},
\end{aligned}
$$

$$
\begin{aligned}
\delta G_{\tilde{H}}^{<}(X, k)= & -\frac{i \pi}{\omega_{\widetilde{H}}}(k+|\mu|)\left\{\delta f_{\widetilde{H}}(X, \vec{k}) \delta\left(k^{0}-\omega_{\widetilde{H}}\right)\right. \\
& \left.+\left[1+\delta \bar{f}_{\widetilde{H}}(X,-\vec{k})\right] \delta\left(k^{0}+\omega_{\tilde{H}}\right)\right\},
\end{aligned}
$$

where we have assumed that the mass term is well approximated by the bare mass term $|\mu|$. Inserting this ansatz into the QBE for the fermionic degrees of freedom (25) one can recover the usual Boltzmann-diffusion equation for the Higgsino number inside the Higgs boson bubble wall once the same approximations discussed in Sec. V B have been made.

\section{THE BARYON NUMBER ASYMMETRY}

Once we have computed the $C P$-violating sources for the right-handed top squark number and the Higgsino number and we have shown that in the thick wall limit the particle number changing interactions in the QBE's reduce to the more familiar Boltzmann-diffusion equations, we are ready to estimate the final baryon asymmetry produced during the electroweak phase transition. From now on we will closely follow the approach taken in Refs. $[7,8]$ where the reader is referred to for more details. If the system is near thermal equilibrium and particles interact weakly, the particle number densities $n_{i}$ may be expressed as $n_{i}=k_{i} \mu_{i} T^{2} / 6$ where $\mu_{i}$ is the local chemical potential, and $k_{i}$ are statistical factors of order of 2 (1) for light bosons (fermions) in thermal equilibrium, and Boltzmann suppressed for particles heavier than $T$.

The particle densities we need to include are the lefthanded top doublet $q_{L} \equiv\left(t_{L}+b_{L}\right)$, the right-handed top quark $t_{R}$, the Higgs particle $H \equiv\left(H_{1}^{0}, H_{2}^{0}, H_{1}^{-}, H_{2}^{+}\right)$, and the superpartners $\widetilde{t}_{R}$ and $\widetilde{H}$-remember that the left-handed top squark and bottom are supposed to be heavier than the temperature $T$. As usual, we shall assume that the supergauge interactions are in equilibrium. Under these assumptions the system may be described by the densities $\mathcal{Q}=q_{L}, \mathcal{T}=t_{R}$ $+\tilde{t}_{R}$, and $\mathcal{H}=H+\widetilde{H}$. Ignoring the curvature of the bubble wall, any quantity becomes a function of the coordinate $\mathbf{z}$ $=z_{3}+v_{\omega} z$, the coordinate normal to the wall surface, where we assume the bubble wall is moving along the $z_{3}$ axis.

Assuming that the rates of the interactions induced by the top Yukawa coupling $\Gamma_{\text {top }}$ and by the strong sphalerons $\Gamma_{\text {ss }}$ are so fast that $\mathcal{Q} / k_{q}-\mathcal{H} / k_{\mathcal{H}}-\mathcal{T} / k_{\mathcal{T}}=\mathcal{O}\left(1 / \Gamma_{\text {top }}\right)$ and $2 \mathcal{Q} / k_{q}$ $-\mathcal{T} k_{\mathcal{T}}+9(\mathcal{Q}+\mathcal{T}) / k_{b}=\mathcal{O}\left(1 / \Gamma_{\mathrm{ss}}\right)$, one can find the equation governing the Higgs density [7]

$$
v_{\omega} \mathcal{H}^{\prime}-\bar{D} \mathcal{H}^{\prime \prime}+\bar{\Gamma} \mathcal{H}-\tilde{\gamma}=0
$$

where the derivatives are now with respect to $\mathbf{z}, \bar{D}$ is the effective diffusion constant, $\tilde{\gamma}$ is an effective source term in the frame of the bubble wall, and $\bar{\Gamma}$ is the effective decay constant [7]. An analytical solution to Eq. (64) satisfying the boundary conditions $\mathcal{H}( \pm \infty)=0$ may be found in the symmetric phase (defined by $\mathbf{z}<0$ ) using a $\mathbf{z}$-independent effective diffusion constant and a step function for the effective decay rate $\bar{\Gamma}=\tilde{\Gamma} \theta(\mathbf{z})$. A more realistic form of $\bar{\Gamma}$ would interpolate smoothly between the symmetric and the broken 
phase values. One can check, however, that the result is insensitive to the specific position of the step function inside the bubble wall [8]. The values of $\bar{D}$ and $\bar{\Gamma}$ in Eq. (64) of course depend on the particular values of supersymmetric parameters. For the considered range we typically find $\bar{D} \sim 0.8 \mathrm{GeV}^{-1}, \bar{\Gamma} \sim 1.7 \mathrm{GeV}[8]$.

The tunneling processes from the symmetric phase to the true minimum in the first order phase transition of the Higgs boson field in the MSSM has been recently analyzed in Ref. [28] including the leading two-loop effects. It was shown that the Higgs boson profile along the bubbles at the time when the latter are formed have a typical thickness $L_{\omega}$ $\sim(20-30) / T .{ }^{6}$ The total amount of the baryon asymmetry is proportional to $\Delta \beta$-the change in the ratio of the Higgs boson vacuum expectation values $\beta=v_{2} / v_{1}$ from $\mathbf{z}=0$ to inside the bubble wall. This quantity tends to zero for large values of $m_{A}$, and takes small values, of order $10^{-2}$ for values of the pseudoscalar mass $m_{A}=150-200 \mathrm{GeV}$ [28].

The dependence of the final baryon asymmetry on $\tilde{\gamma}$ is much more delicate. The solution of Eq. (64) for $\mathbf{z}<0$ is

$$
\mathcal{H}(\mathbf{z})=\mathcal{A} e^{\mathbf{z} \omega_{\omega} / \bar{D}},
$$

and for $\mathbf{z}>0$,

$$
\begin{aligned}
\mathcal{H}(\mathbf{z})= & \left(\mathcal{B}_{+}-\frac{1}{\bar{D}\left(\lambda_{+}-\lambda_{-}\right)} \int_{0}^{\mathbf{z}} d u \tilde{\gamma}(u) e^{-\lambda_{+} u}\right) e^{\lambda_{+} \mathbf{z}} \\
& +\left(\mathcal{B}_{-}-\frac{1}{\bar{D}\left(\lambda_{-}-\lambda_{+}\right)} \int_{0}^{\mathbf{z}} d u \tilde{\gamma}(u) e^{-\lambda_{-} u}\right) e^{\lambda_{-} \mathbf{z}},
\end{aligned}
$$

where

$$
\lambda_{ \pm}=\frac{v_{\omega} \pm \sqrt{v_{\omega}^{2}+4 \tilde{\Gamma} \bar{D}}}{2 \bar{D}}
$$

and $\tilde{\gamma}(\mathbf{z})$ is the total $C P$-violating current resulting from the sum of the right-handed top squark and Higgsino contributions. Imposing the continuity of $\mathcal{H}$ and $\mathcal{H}^{\prime}$ at the boundaries, we find

\footnotetext{
${ }^{6}$ In general, however, the value of $L_{\omega}$ when the bubbles are moving through the plasma with some velocity $v_{\omega}$ is different from the value at bubble nucleation. Indeed, the motion of the bubble wall is determined by two main factors, namely, the pressure difference between inside and outside the bubble-leading to the expansionand the friction force, proportional to $v_{\omega}$, accounting for the collisions of the plasma particles off the wall. The equilibrium between these two forces imples a steady state with a final velocity $v_{\omega}$. If bubbles are rather thick, thermodynamical conditions are established inside the wall and for the latter it is no longer possible to lose energy by thermal dissipation. Under these conditions the bubble wall is accelerated until slightly out-of-equilibrium conditions and the friction forces are reestablished.
}

$\mathcal{A}=\mathcal{B}_{+}\left(1-\frac{\lambda_{-}}{\lambda_{+}}\right)=\mathcal{B}_{-}\left(\frac{\lambda_{+}}{\lambda_{-}}-1\right)=\frac{1}{\bar{D} \lambda_{+}} \int_{0}^{\infty} d u \tilde{\gamma}(u) e^{-\lambda_{+} u}$

From the form of the above equations one can see that $C P$-violating densities are nonzero for a time $t \sim \bar{D} / v_{\omega}^{2}$ and the assumptions leading to the analytical form of $\mathcal{H}(\mathbf{z})$ from Eq. (64) - are valid provided that the interaction rates $\Gamma_{\text {top }}$ and $\Gamma_{\mathrm{ss}}$ are larger than $v_{\omega}^{2} / \bar{D}[7,8]$.

The equation governing the baryon asymmetry $n_{B}$ is given by [7]

$$
D_{q} n_{B}^{\prime \prime}-v_{\omega} n_{B}^{\prime}-\theta(-\mathbf{z}) n_{f} \Gamma_{\mathrm{ws}} n_{L}=0,
$$

where $\Gamma_{\mathrm{ws}}=6 \kappa \alpha_{w}^{4} T$ is the weak sphaleron rate $(\kappa \simeq 1)[29]$ (the correct value of $\kappa$ is at present the subject of debate), and $n_{L}$ is the total number density of left-handed weak doublet fermions, $n_{f}=3$ is the number of families, and we have assumed that the baryon asymmetry gets produced only in the symmetric phase. Expressing $n_{L}(\mathbf{z})$ in terms of the Higgs number density

$$
n_{L}=\frac{9 k_{q} k_{\mathcal{T}}-8 k_{b} k_{\mathcal{T}}-5 k_{b} k_{q}}{k_{\mathcal{H}}\left(k_{b}+9 k_{q}+9 k_{\mathcal{T}}\right)} \mathcal{H}
$$

and making use of Eqs. (65)-(69), we find that

$$
\frac{n_{B}}{s}=-g\left(k_{i}\right) \frac{\mathcal{A} \bar{D} \Gamma_{\mathrm{ws}}}{v_{\omega}^{2} s},
$$

where $s=2 \pi^{2} g_{* s} T^{3} / 45$ is the entropy density ( $g_{* s}$ being the effective number of relativistic degrees of freedom) and $g\left(k_{i}\right)$ is a numerical coefficient depending upon the light degrees of freedom present in the thermal bath.

We see that the final baryon asymmetry depends sensitively on the parameter $\mathcal{A}$, that is, on the integral of the source. In a previous analysis $[7,8]$ the $C P$-violating sources were inserted into the diffusion equations by hand only after the $C P$-violating currents were defined and computed. More specifically, $C P$-violating sources $\mathcal{S}$ associated to a generic charge density $j^{0}$ have been constructed from the current $j^{\mu}$ by the definition $\mathcal{S}=\partial_{0} j^{0}[7,8]$. This procedure has introduced an unacceptable degree of arbitrariness-different definitions of $C P$-violating currents $j^{\mu}$ have been adopted in the literature $[7,8]$ and have lead to different numerical results for the final baryon asymmetry. This is because the corresponding sources differ in terms of number of derivatives of the Higgs boson bubble wall profile and, therefore, in terms of different powers of the bubble wall velocity $v_{\omega}$ and bubble wall width $L_{\omega}$.

The basic merit of the CTP formalism is that it provides us with a rigorous and self-consistent definition of the $C P$-violating sources within the quantum Boltzmann equations [15]. The computation of the $C P$-violating currents for the right-handed top squark and Higgsino local densities was performed in Ref. [8] by means of the CTP formalism. Since currents were proportional to $\dot{\beta}$ in the thick bubble wall limit, i.e., proportional to the first time derivative of the 
Higgs boson profile, sources turned out to be proportional to the second time derivative of the Higgs boson profile [8]. However, the derivation of the transport QBE's allows us to define the $C P$-violating sources uniquely, since they are automatically incorporated in the right-hand side of the QBE's [15]. This self-consistent procedure indicates that the sources in the quantum diffusion equations are proportional to the first time derivative of the Higgs boson configuration. A comparison between the sources $\mathcal{S}_{\tilde{t}_{R}}$, see Eq. (38), and $\mathcal{S}_{\tilde{H}}$, see Eq. (61), obtained in the present work and the currents $j^{0}$ given in Ref. [8] indicate that they may be related as

$$
\mathcal{S}(T) \sim \frac{j^{0}(T)}{\tau}
$$

and it may be interpreted as the time derivative of the current density accumulated at the time $T$ after the wall has deposited at a given specific point the current density $j^{0}$ each interval $\tau$

$$
\mathcal{S}(T) \sim \partial_{0} \int^{T} d t \frac{j^{0}(t)}{\tau}
$$

Here $\tau=\Gamma^{-1}$ is the thermalization time of the right-handed top squarks and Higgsinos, respectively. The integral over time is peculiar of the quantum approach and it induces memory effects.

The parameter $\mathcal{A}$ computed from the sources (38) and (61) and rigorously derived from the QBE's turns out to be ${ }^{7}$

$$
\begin{gathered}
\mathcal{A}=\frac{2 f\left(k_{i}\right) \Gamma_{\widetilde{H}}}{\bar{D} \lambda_{+}} \int_{0}^{\infty} d u j_{\widetilde{H}}^{0}(u) e^{-\lambda_{+} u} \propto \frac{2 f\left(k_{i}\right) \Gamma_{\widetilde{H}}}{\bar{D} \lambda_{+}} I, \\
I \equiv \int_{0}^{\infty} d u v^{2}(u) \frac{d \beta(u)}{d u} e^{-\lambda_{+} u} \simeq \int_{0}^{\infty} d u v^{2}(u) \frac{d \beta(u)}{d u},
\end{gathered}
$$

where $j_{\widetilde{H}}^{0}$ is the Higgsino current computed in Ref. [8], $\Gamma_{\widetilde{H}}$ is the Higgsino damping rate, and $f\left(k_{i}\right)$ is a coefficient depending upon the number of degrees of freedom present in the thermal bath.

A comparison between this parameter $\mathcal{A}$ and the one obtained in Ref. [8] indicates that memory effects introduce an enhancement factor in the final baryon asymmetry

$$
\frac{2 \Gamma}{\lambda_{+} v_{\omega}} \simeq 70\left(\frac{\Gamma_{\tilde{H}}}{5 \times 10^{-2} T}\right)\left(\frac{0.1}{v_{\omega}}\right)
$$

${ }^{7}$ For large values of the left-handed top squark mass the top squark contribution to the baryon asymmetry is strongly suppressed compared to the chargino and neutralino ones and the numerical values of the baryon asymmetry depend linearly on the phase of the Higgsino mass parameter. with respect to the results given in Ref. [8]. ${ }^{8}$ Our results indicate that memory effects play a crucial role in relaxing the stringent lower bound on the values of the $C P$-violating phases obtained in previous analyses $[8,10]$. In particular, the observed baryon asymmetry $n_{B} / s \simeq 4 \times 10^{-11}$ may be originated by Higgsinos if the $C P$-violating phase $\phi_{\mu}$ of the $\mu$ parameter is

$$
\left|\sin \left(\phi_{\mu}\right)\right| \geq 10^{-3}\left(\frac{v_{\omega}}{0.1}\right)
$$

This lower bound is two orders of magnitude weaker than the one obtained in previous works $[8,10]$. These small values of the phase $\phi_{\mu}$ are consistent with the constraints from the electric dipole moment of the neutron even if the squarks of the first and second generation have masses of the order of $100 \mathrm{GeV}$. In the limit of thick bubble walls, the $C P$-violating sources are also characterized by resonance effects $[8,15]$ when the particles involved in the construction of the source are degenerate in mass. The resonance is manifest in the function $G$ defined in Eq. (40). The interpretation of the resonance is rather straightforward if we think in terms of scatterings of the quasiparticles off the advancing low momentum bubble wall configuration. For momenta of order of the critical temperature, the scattering is more efficient when the Higgsinos and gauginos are nearly degenerate in mass, $\mu \sim M_{2}$. A similar effect has been found in Ref. [10], where the system was studied in the classical limit. These classical treatments somehow obscure the origin of the $C P$-violating effects as resulting from quantum interference and the origin of the resonance. Moreover, memory effects are not present in the classical approach which, therefore, underestimates the value of the final baryon asymmetry.

\section{CONCLUSIONS}

In conclusion, we have reanalyzed the computation of the baryon asymmetry generated at the electroweak phase transition in the minimal supersymmetric standard model. We have argued that the CTP formalism is the natural guide towards a rigorous and self-consistent definition of the $C P$-violating sources within the quantum Boltzmann equa-

\footnotetext{
${ }^{8}$ The integral $I$ has been computed including two-loop effects in Ref. [28] and turns out to be $I \simeq 10^{-2}$ for $m_{A}=150-200 \mathrm{GeV}$. The same value (in absolute value) of the integral was obtained in Ref. [8] by making use of a reasonable ansatz for the Higgs profile. The reason is that this result was based on the temperature $T_{0}$ defined to be the temperature at which the Higgs potential becomes flat at the origin. This temperature is lower than the temperature $T_{c}$ at which the tunneling process occurs and the numerical result of Ref. [28] indicates that the integral $I$ is a decreasing function of the temperature. Since the results of Ref. [8] were based on the one-loop effective potential, the result in Ref. [28] is accidentally very close to the numerical result of Ref. [8]. Therefore, the numerical difference between the value of the final baryon asymmetry obtained here and the one evaluated in Ref. [8] reduces to the expression (75). We thank M. Quiros for discussions on this point.
} 
tions. The QBE's manifest-in the scattering terms-nonMarkovian features. In the limit of the thick bubble wall, however, a number of approximations may be performed so that the QBE's get reduced to the more familiar diffusionBoltzmann equations. Strong memory effects enhance the strength of the $C P$-violating sources by at least two orders of magnitude. The baryon asymmetry is mainly generated by Higgsinos, provided that Higgsinos and gauginos are not much heavier than the electroweak critical temperature and the phase $\phi_{\mu}$ is larger than $10^{-3}$. This value is further re- duced for $v_{\omega} \lesssim 0.1$. It is intriguing that these small values of the phases are perfectly consistent with the constraints from the electric dipole moment of the neutron and squarks of the first and second generations as light as $\sim 100 \mathrm{GeV}$ may be tolerated.

\section{ACKNOWLEDGMENTS}

The author would like to thank M. Carena, M. Quiros, and C. E. M. Wagner for useful discussions.
[1] For recent reviews, see A. G. Cohen, D. B. Kaplan, and A. E. Nelson, Annu. Rev. Nucl. Part. Sci. 43, 27 (1993); M. Quirós, Helv. Phys. Acta 67, 451 (1994); V. A. Rubakov and M. E. Shaposhnikov, Usp. Fiz. Nauk 166, 493 (1996) [Phys. Usp. 39, 461 (1996)].

[2] G. 't Hooft, Phys. Rev. Lett. 37, 8 (1976); Phys. Rev. D 14, 3432 (1976); P. Arnold and L. McLerran, ibid. 36, 581 (1987); 37, 1020 (1988); S. Yu Khlebnikov and M. E. Shaposhnikov, Nucl. Phys. B308, 885 (1988); F. R. Klinkhamer and N. S. Manton, Phys. Rev. D 30, 2212 (1984); B. Kastening, R. D. Peccei, and X. Zhang, Phys. Lett. B 266, 413 (1991); L. Carson, Xu Li, L. McLerran, and R.-T. Wang, Phys. Rev. D 42, 2127 (1990); M. Dine, P. Huet, and R. Singleton Jr., Nucl. Phys. B375, 625 (1992).

[3] M. Shaposhnikov, JETP Lett. 44, 465 (1986); Nucl. Phys. B287, 757 (1987); B299, 797 (1988); M. E. Carrington, Phys. Rev. D 45, 2933 (1992); M. Dine, R. G. Leigh, P. Huet, A. Linde, and D. Linde, Phys. Lett. B 283, 319 (1992); Phys. Rev. D 46, 550 (1992); P. Arnold, ibid. 46, 2628 (1992); J. R. Espinosa, M. Quirós, and F. Zwirner, Phys. Lett. B 314, 206 (1993); W. Buchmüller, Z. Fodor, T. Helbig, and D. Walliser, Ann. Phys. (N.Y.) 234, 260 (1994); J. Bagnasco and M. Dine, Phys. Lett. B 303, 308 (1993); P. Arnold and O. Espinosa, Phys. Rev. D 47, 3546 (1993); Z. Fodor and A. Hebecker, Nucl. Phys. B432, 127 (1994); K. Kajantie, K. Rummukainen, and M. E. Shaposhnikov, ibid. B407, 356 (1993); Z. Fodor, J. Hein, K. Jansen, A. Jaster, and I. Montvay, ibid. B439, 147 (1995); K. Kajantie, M. Laine, K. Rummukainen, and M. E. Shaposhnikov, ibid. B466, 189 (1996).

[4] G. F. Giudice, Phys. Rev. D 45, 3177 (1992).

[5] S. Myint, Phys. Lett. B 287, 325 (1992).

[6] J. R. Espinosa, M. Quirós, and F. Zwirner, Phys. Lett. B 307, 106 (1993); A. Brignole, J. R. Espinosa, M. Quirós, and F. Zwirner, ibid. 324, 181 (1994).

[7] P. Huet and A. E. Nelson, Phys. Rev. D 53, 4578 (1996).

[8] M. Carena, M. Quiros, A. Riotto, I. Vilja, and C. E. M. Wagner, Nucl. Phys. B503, 387 (1997).
[9] A. Riotto, Repport No. OUTP-97-43-P, hep-ph/9709286.

[10] J. M. Cline, M. Joyce, and K. Kainulainen, Phys. Lett. B 417, 79 (1998).

[11] M. Carena, M. Quiros, and C. E. M. Wagner, Phys. Lett. B 380, 81 (1996); D. Delepine, J. M. Gerard, R. Gonzalez Felipe, and J. Weyers, ibid. 386, 183 (1996); J. R. Espinosa, Nucl. Phys. B475, 273 (1996); B. de Carlos and J. R. Espinosa, ibid. B503, 24 (1997).

[12] M. Carena, M. Quiros, and C. E. M. Wagner, Nucl. Phys. B524, 3 (1998).

[13] M. Laine, Nucl. Phys. B481, 43 (1996); J. M. Cline and K. Kainulainen, ibid. B482, 73 (1996).

[14] M. Joyce, T. Prokopec, and N. Turok, Phys. Lett. B 339, 312 (1994); A. G. Cohen, D. B. Kaplan, and A. E. Nelson, ibid. 336, 41 (1994).

[15] A. Riotto, Nucl. Phys. B518, 339 (1998).

[16] P. Danielewicz, Ann. Phys. (N.Y.) 152, 239 (1984); 152, 305 (1984).

[17] A. Riotto, Phys. Rev. D 53, 5834 (1996).

[18] P. A. Henning, Phys. Rep. 253, 235 (1995).

[19] J. Schwinger, J. Math. Phys. 2, 407 (1961); L. V. Keldysh, JETP Lett. 20, 1018 (1965).

[20] K. Chou, Z. Su, B. Hao, and L. Yu, Phys. Rep. 118, 1 (1985), and references therein.

[21] R. A. Craig, J. Math. Phys. 9, 605 (1968).

[22] L. P. Kadanoff and G. Baym, Quantum Statistical Mechanics (Benjamin, New York, 1962).

[23] K. Enqvist, A. Riotto, and I. Vilja, Report No. OUTP-97-49-P, hep-ph/9710373.

[24] V. Spicka and P. Lipavsky, Phys. Rev. Lett. 73, 3439 (1994).

[25] H. A. Weldon, Phys. Rev. D 26, 1394 (1982); V. V. Klimov, Sov. Phys. JETP 55, 199 (1982).

[26] A. Riotto and I. Vilja, Phys. Lett. B 402, 314 (1997); O. K. Kalashnikov, JETP Lett. 67, 1 (1998).

[27] P. Elmfors, K. Enqvist, A. Riotto, and I. Vilja (in preparation).

[28] J. M. Moreno, M. Quiros, and M. Seco, Report No. IEM-FT168/98, hep-ph/9801272.

[29] J. Ambjorn and A. Krasnitz, Phys. Lett. B 362, 97 (1995). 